\title{
单一金属氧化物同时催化去除碳颗粒和 $\mathrm{NO}_{x}$
}

\author{
朱荣淑*郭明新欧阳峰* \\ (哈尔滨工业大学深圳研究生院环境科学与工程研究中心, 广东 深圳 518055)
}

\begin{abstract}
摘要：制备了系列金属氧化物催化剂, 研究了富氧条件下单一金属氧化物同时催化去除碳颗粒和 $\mathrm{NO}_{x}$ 的活 性, 考察了碳颗粒与催化剂之间的接触方式对催化活性的影响, 并分析了碳颗粒和 $\mathrm{NO}_{x}$ 催化同时去除的路径. 结果表明, $\mathrm{Cr} 、 \mathrm{Mn} 、 \mathrm{Co}$ 和 $\mathrm{Ni}$ 金属氧化物催化剂对碳颗粒和 $\mathrm{NO}_{x}$ 同时去除具有较高的催化活性, 并且在催化剂 与碳颗粒之间 “松散接触”方式下依然具有较高的活性; 其同时催化去除碳颗粒和 $\mathrm{NO}_{x}$ 的路径为, 催化剂催化 $\mathrm{NO}$ 氧化成 $\mathrm{NO}_{2}, \mathrm{NO}_{2}$ 促进碳颗粒氧化去除, 而碳颗粒氧化的中间物 $\mathrm{CO}$ 还原 $\mathrm{NO}$, 促进 $\mathrm{NO}_{x}$ 还原去除.
\end{abstract}

关键词：金属氧化物; 同时催化去除; 碳颗粒; NO

中图分类号: $\mathrm{O} 643$

\section{Simultaneous Catalytic Removal of Soot and $\mathrm{NO}_{x}$ over Single Metal Oxide Catalysts}

\author{
ZHU Rong-Shu* GUO Ming-Xin OUYANG Feng * \\ (Environmental Science and Engineering Research Center, Harbin Institute of Technology Shenzhen Graduate School, \\ Shenzhen 518055, Guangdong Province, P. R. China)
}

\begin{abstract}
A series of single metal oxide catalysts were prepared and their catalytic activities were investigated for simultaneous removal of soot and $\mathrm{NO}_{x}$ in an oxygen rich condition. Effects of contact intensity between catalyst and soot as well as simultaneous removal paths for soot and $\mathrm{NO}_{x}$ were also studied. Results showed that metal oxide catalysts of $\mathrm{Cr}, \mathrm{Mn}, \mathrm{Co}$, and $\mathrm{Ni}$ exhibited high activities for simultaneous removal of soot and $\mathrm{NO}_{x}$ and still had high activities in the loose contact mode between catalyst and soot. Simultaneous removal paths found were that $\mathrm{NO}_{2}$ formation by oxidation of $\mathrm{NO}$ promoted soot oxidation and $\mathrm{CO}$ formation by soot oxidation reduced $\mathrm{NO}_{x}$.
\end{abstract}

Key Words: Metal oxide; Simultaneous catalytic removal; Soot; NO

自 Yoshida 等 ${ }^{[1]}$ 提出“soot- $\mathrm{O}_{2}$-NO”三组分之间 的反应以来, 用柴油机过滤器上收集的碳颗粒还原 氮氧化物 $\left(\mathrm{NO}_{x}\right)$ 引起了人们的普遍关注 ${ }^{[2-12]}$. 利用过 滤器上收集的碳颗粒还原 $\mathrm{NO}_{x}$ 是“过滤器捕集颗粒 物-碳颗粒还原 $\mathrm{NO}_{x}$-过滤器再生”一体化的新技术路 线, 是实现同时去除柴油机尾气中碳颗粒和 $\mathrm{NO}_{x}$ 最 理想的技术途径. Shangguan 等 ${ }^{[2,3,7,100}$ 对同时催化去 除柴油机尾气中碳颗粒和 $\mathrm{NO}_{x}$ 进行了一系列基础 性研究, 发现了钻钛矿型和尖晶石型复合氧化物催
化体系对同时去除碳颗粒和 $\mathrm{NO}_{x}$ 具有较高的催化 活性. 然而, 对单一金属氧化物催化同时去除碳颗 粒和 $\mathrm{NO}_{x}$ 的研究关注较少. 另外, Neeft 等 ${ }^{[13]}$ 研究了 金属氧化物催化剂对碳颗粒催化氧化的活性, 发现 了催化剂与碳颗粒之间的接触方式对碳颗粒的催 化氧化活性影响很大, 同一催化剂与碳颗粒在“紧密 接触”时的催化活性总比在“松散接触”时的催化活 性大. 然而, 在大多数已报道的关于碳颗粒和 $\mathrm{NO}_{x}$ 同时催化去除的研究中都采用催化剂与碳颗粒“紧

Received: July 25, 2008; Revised: October 16, 2008; Published on Web: November 26, 2008.

*Corresponding authors. Email: ouyangfh@hit.edu.cn, rszhu@hitsz.edu.cn; Tel: +86755-26032557.

哈尔滨工业大学科研创新基金资助项目

C. Editorial office of Acta Physico-Chimica Sinica 
密接触”的方式. 接触方式对催化活性的影响也很少 被关注.

在本研究中, 对单一金属氧化物催化同时去除 碳颗粒和 $\mathrm{NO}_{x}$ 进行了研究, 目的是研究单一金属 氧化物催化剂对碳颗粒和 $\mathrm{NO}_{x}$ 同时去除的催化活 性以及金属氧化物催化剂与碳颗粒之间的接触方 式对催化活性的影响, 同时也对碳颗粒和 $\mathrm{NO}_{x}$ 同 时去除的路径进行了分析.

\section{1 实验部分}

\section{1 催化剂的制备}

本研究所用的催化剂都为无负载型金属氧化 物. 所有催化剂都是将前驱物在空气中 $600{ }^{\circ} \mathrm{C}$ 焙烧 $3 \mathrm{~h}$ 制得. 金属氧化物催化剂前驱物为金属盐或金 属氧化物(上海国药集团化学试剂有限公司), 均为 分析纯, 详细清单见表 1 .

\section{2 催化剂活性评价}

催化剂活性通过程序升温反应(TPR)技术进行 评价. 评价实验在连续流动固定床反应装置上进 行 ${ }^{[14]}$. 反应器为内径 $6 \mathrm{~mm}$ 的石英管. 实验所需温度 由单管电阻炉提供, 其温度由可编程温控仪(北京朝 阳自动化仪表厂, CKW-2200)控制, 程序升温速率为 $4{ }^{\circ} \mathrm{C} \cdot \mathrm{min}^{-1}$. 分析检测系统由气相色谱仪和 $\mathrm{NO}_{x}$ 分 析仪构成, 反应过程中产生的 $\mathrm{CO}$ 和 $\mathrm{CO}_{2}$ 的浓度由 配有氢火焰离子化检测器(FID)的气相色谱仪(中国 上海精密科学仪器有限公司, GC-112A)测定, NO、 $\mathrm{NO}_{2} 、 \mathrm{NO}_{x}\left(\mathrm{NO}_{x}=\mathrm{NO}+\mathrm{NO}_{2}\right)$ 浓度由 $\mathrm{NO}_{x}$ 分析仪(澳大 利亚 ECOTECH 公司, EC9841)测定. 气体检测均为 在线检测.

本研究采用由德国 Degussa 公司提供的 Printex-U 碳颗粒模拟柴油机排放的碳颗粒, 其具

表 1 各种金属氧化物催化剂及其前驱物

Table 1 Metal oxide catalysts and their precursor

\begin{tabular}{rccccc}
\hline No. & Catalyst & Precursor & No. Catalyst & Precursor \\
\hline 1 & $\mathrm{~V}_{2} \mathrm{O}_{5}$ & $\mathrm{NH}_{4} \mathrm{VO}_{4}$ & 13 & $\mathrm{Ce}_{2} \mathrm{O}_{3}$ & $\mathrm{Ce}\left(\mathrm{NO}_{3}\right)_{3} \cdot 6 \mathrm{H}_{2} \mathrm{O}$ \\
2 & $\mathrm{WO}_{3}$ & $\left(\mathrm{NH}_{4}\right)_{10} \mathrm{~W}_{12} \mathrm{O}_{41}$ & 14 & $\mathrm{MnO}_{2}$ & $\mathrm{Mn}\left(\mathrm{NO}_{3}\right)_{2}$ \\
3 & $\mathrm{MoO}_{3}$ & $\left(\mathrm{NH}_{4}\right)_{6} \mathrm{Mo}_{7} \mathrm{O}_{24} \cdot 4 \mathrm{H}_{2} \mathrm{O}$ & 15 & $\mathrm{Co}_{3} \mathrm{O}_{4}$ & $\mathrm{Co}\left(\mathrm{NO}_{3}\right)_{2} \cdot 6 \mathrm{H}_{2} \mathrm{O}$ \\
4 & $\mathrm{Cr}_{2} \mathrm{O}_{3}$ & $\left(\mathrm{NH}_{4}\right)_{2} \mathrm{Cr}_{2} \mathrm{O}_{7}$ & 16 & $\mathrm{CuO}$ & $\mathrm{Cu}\left(\mathrm{NO}_{3}\right)_{2} \cdot 3 \mathrm{H}_{2} \mathrm{O}$ \\
5 & $\mathrm{SnO}_{2}$ & $\mathrm{SnSO}_{4}$ & 17 & $\mathrm{Bi}_{2} \mathrm{O}_{3}$ & $\mathrm{Bi} \mathrm{Bi}_{3} \mathrm{O}(\mathrm{OH})_{9}\left(\mathrm{NO}_{3}\right)_{4}$ \\
6 & $\mathrm{ZnO}$ & $\mathrm{ZnSO}_{4} \cdot 7 \mathrm{H}_{2} \mathrm{O}$ & 18 & $\mathrm{Sb}_{2} \mathrm{O}_{3}$ & $\mathrm{Sb}_{2} \mathrm{O}_{3}$ \\
7 & $\mathrm{Fe}_{2} \mathrm{O}_{3}$ & $\mathrm{Fe}\left(\mathrm{NO}_{3}\right)_{3} \cdot 9 \mathrm{H}_{2} \mathrm{O}$ & 19 & $\mathrm{TiO}_{2}$ & $\mathrm{TiO}_{2}$ \\
8 & $\mathrm{NiO}$ & $\mathrm{Ni}\left(\mathrm{NO}_{3}\right)_{2} \cdot 6 \mathrm{H}_{2} \mathrm{O}$ & 20 & $\mathrm{Al}_{2} \mathrm{O}_{3}$ & $\mathrm{Al}_{2} \mathrm{O}_{3}$ \\
9 & $\mathrm{CdO}$ & $\mathrm{Cd}\left(\mathrm{NO}_{3}\right)_{2}$ & 21 & $\mathrm{Nb}_{2} \mathrm{O}_{5}$ & $\mathrm{Nb}_{2} \mathrm{O}_{5}$ \\
10 & $\mathrm{PbO}$ & ${\mathrm{Pb}\left(\mathrm{NO}_{3}\right)_{2}}_{22}$ & $\mathrm{Ta}_{2} \mathrm{O}_{5}$ & $\mathrm{Ta}_{2} \mathrm{O}_{5}$ \\
11 & $\mathrm{In}_{2} \mathrm{O}_{3}$ & $\mathrm{In}\left(\mathrm{NO}_{3}\right)_{3}$ & 23 & $\mathrm{Ga}_{2} \mathrm{O}_{5}$ & $\mathrm{Ga}_{2} \mathrm{O}_{5}$ \\
12 & $\mathrm{ZrO}_{2}$ & $\mathrm{Zr}\left(\mathrm{NO}_{3}\right)_{4} \cdot 5 \mathrm{H}_{2} \mathrm{O}$ & 24 & $\mathrm{Ge}_{2} \mathrm{O}_{5}$ & $\mathrm{Ge}_{2} \mathrm{O}_{5}$ \\
\hline
\end{tabular}

体组分含量与文献[13]相同. 本评价实验包括催化 剂与碳颗粒“紧密接触”和“松散接触”两种方式. “紧密接触”: 将碳颗粒与催化剂按 1:10 质量比混 合后置于玛瑙研钵中研磨 $1 \mathrm{~h}$, 然后在 $20 \mathrm{MPa}$ 压 力下压片, 经破碎后篮分出粒径为 40-60 目(0.125$0.425 \mathrm{~mm}$ ) 的样品颗粒以备实验所用; “松散接触”: 将粉末催化剂在 $20 \mathrm{MPa}$ 压力下压片, 经破碎后篮 分出粒径为 40-60 目的催化剂颗粒, 然后用药匙将 碳颗粒与已篮分好的粒径为 40-60 目的催化剂按 1:10 质量比混合. 空白实验使用粒径为 40-60 目的 $\mathrm{SiO}_{2}$ 微球颗粒进行. 每次实验取总重量为 $0.055 \mathrm{~g}$ (催化剂 $0.05 \mathrm{~g}$, 碳颗粒 $0.005 \mathrm{~g}$ ) 的样品置于反应器 中. 反应混合气的体积分数组成为 $\mathrm{NO}(0.042 \%) 、 \mathrm{O}_{2}$ $(4.4 \%) 、 \mathrm{He}$ (平衡气), 气体总流量为 $100 \mathrm{~mL} \cdot \mathrm{min}^{-1}$. 部分实验中反应气组分改变, 但各反应气浓度保持 不变, 气体总流量保持不变.

催化剂对碳颗粒和 $\mathrm{NO}_{x}$ 同时去除的催化活性, 主要可由两个参数来评价: 碳颗粒总量被氧化 $10 \%$ 时的温度 $\left(T_{10},{ }^{\circ} \mathrm{C}\right)$ 和一个 TPR 过程中 $\mathrm{NO}_{x}$ 减少 总量 $\left(V_{\mathrm{deNO}}, \mathrm{mol}\right) . T_{10}$ 由初始形成碳氧化物 $\mathrm{CO}_{x}\left(\mathrm{CO}_{x}=\right.$ $\mathrm{CO}+\mathrm{CO}_{2}$ ) 量占整个 TPR 过程中形成总 $\mathrm{CO}_{x}$ 量的 $10 \%$ 时对应的温度来确定. $V_{\text {deNO }}$ 可通过将 $\mathrm{NO}_{x}$ 减少量对 温度(时间)坐标积分得到. $T_{10}$ 越低, $V_{\mathrm{deNO}_{x}}$ 越大, 催化 剂活性越高.

\section{2 结果与讨论}

2.1 同时去除碳颗粒和 $\mathrm{NO}_{x}$ 的催化活性

图 1 为 $\mathrm{Cr}_{2} \mathrm{O}_{3}$ 与碳颗粒在 “松散接触”方式下

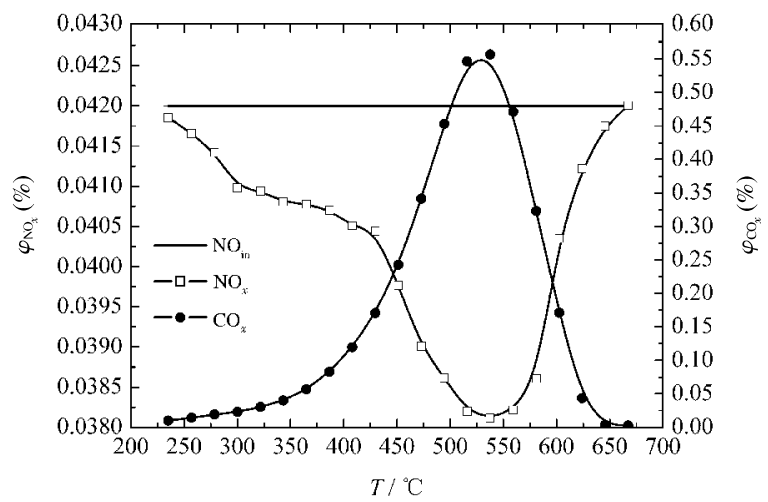

图 $1 \mathrm{Cr}_{2} \mathrm{O}_{3}$ 与碳颗粒在“松散接触”方式下进行 $\mathrm{C}+\mathrm{NO}+\mathrm{O}_{2}$ 反应时 $\mathrm{CO}_{x}$ 和 $\mathrm{NO}_{x}$ 的 TPR 曲线

Fig.1 TPR curves of $\mathrm{CO}_{x}$ and $\mathrm{NO}_{x}$ over $\mathrm{Cr}_{2} \mathrm{O}_{3}$ catalyst in loose contact mode

catalyst mass: $0.05 \mathrm{~g}$, soot mass: $0.005 \mathrm{~g}$; reactant conditions: $\mathrm{C}+0.042 \% \mathrm{NO}+4.4 \% \mathrm{O}_{2}+\mathrm{He}$, flow rate: $100 \mathrm{~mL} \cdot \mathrm{min}^{-1}$; $\mathrm{NO}_{\text {in }}$ is the concentration of inlet $\mathrm{NO}$ 
进行 $\mathrm{C}+\mathrm{NO}+\mathrm{O}_{2}$ 反应时 $\mathrm{CO}_{x}$ 和 $\mathrm{NO}_{x}$ 的 $\mathrm{TPR}$ 曲线. 从图 1 中可以看到, 碳颗粒的氧化和 $\mathrm{NO}_{x}$ 的减少 发生在同一温度范围内, 这表明发生了碳颗粒和 $\mathrm{NO}_{x}$ 同时去除. 在温度高于 $538{ }^{\circ} \mathrm{C}$ 后, $\mathrm{CO}_{x}$ 浓度出 现下降而 $\mathrm{NO}_{x}$ 浓度出现上升, 这主要是由于碳颗 粒逐渐被耗尽.

表 2 列出了各催化剂与碳颗粒分别在“紧密接 触”和“松散接触”方式下进行 $\mathrm{C}+\mathrm{NO}+\mathrm{O}_{2}$ 反应时的 $T_{10}$ 和 $V_{\mathrm{deNO}}$, 图 2 展示了碳颗粒和 $\mathrm{NO}_{x}$ 同时去除时 $T_{10}$ 和 $V_{\mathrm{deNO} x}$ 的相关性. 从图 2 中可以看到: (1) $\mathrm{PbO}$ 、 $\mathrm{Ce}_{2} \mathrm{O}_{3} 、 \mathrm{~V}_{2} \mathrm{O}_{5} 、 \mathrm{MoO}_{3}$ 在 “紧密接触”方式下具有较低 的 $T_{10}$ 和较小的 $V_{\text {deNO }} ;(2) \mathrm{Al}_{2} \mathrm{O}_{3} 、 \mathrm{Ga}_{2} \mathrm{O}_{5} 、 \mathrm{SnO}_{2}$ 具有较 高的 $T_{10}$ 和较大的 $V_{\text {deNO }} ;(3) \mathrm{Cr}_{2} \mathrm{O}_{3} 、 \mathrm{MnO}_{2} 、 \mathrm{Co}_{3} \mathrm{O}_{4} 、 \mathrm{NiO}$ 具有相对较低的 $T_{10}$ 和相对较大的 $V_{\mathrm{deNO}} ;$; (4) 其余金 属氧化物具有相对较高的 $T_{10}$ 和相对较小的 $V_{\mathrm{deNO}}$. 根据碳颗粒氧化的 $T_{10}$ 越低、 $\mathrm{NO}_{x}$ 减少的 $V_{\mathrm{deNO}}$ 越大、 催化剂活性越高的原则, 那么, $\mathrm{Cr} 、 \mathrm{Mn} 、 \mathrm{Co}$ 和 $\mathrm{Ni}$ 的

\section{表 2 各催化剂与碳颗粒在“紧密接触”和“松散接触”方式 下同时去除碳颗粒和 $\mathrm{NO}_{x}$ 的活性}

Table 2 Activity of catalysts in tight and loose contact for simultaneous removal of soot and $\mathrm{NO}_{x}$

\begin{tabular}{|c|c|c|c|c|}
\hline \multirow{2}{*}{ Catalyst } & \multicolumn{2}{|c|}{ Tight contact } & \multicolumn{2}{|c|}{ Loose contact } \\
\hline & $T_{10}{ }^{\circ} \mathrm{C}$ & $10^{5} V_{\text {deNo }} / \mathrm{mol}$ & $T_{10}{ }^{\circ} \mathrm{C}$ & $10^{5} V_{\mathrm{deNO}} / \mathrm{mol}$ \\
\hline $\mathrm{V}_{2} \mathrm{O}_{5}$ & 382 & 0.50 & 525 & 0.72 \\
\hline $\mathrm{WO}_{3}$ & 496 & 0.86 & 538 & 1.80 \\
\hline $\mathrm{MoO}_{3}$ & 412 & 0.21 & 475 & 0.63 \\
\hline $\mathrm{Cr}_{2} \mathrm{O}_{3}$ & 354 & 2.90 & 410 & 3.25 \\
\hline $\mathrm{SnO}_{2}$ & 519 & 2.55 & 536 & 2.12 \\
\hline $\mathrm{ZnO}$ & 526 & 1.24 & 531 & 1.34 \\
\hline $\mathrm{Fe}_{2} \mathrm{O}_{3}$ & 409 & 0.83 & 497 & 2.03 \\
\hline $\mathrm{NiO}$ & 424 & 2.44 & 473 & 2.45 \\
\hline $\mathrm{CdO}$ & 429 & 1.43 & 501 & 2.22 \\
\hline $\mathrm{PbO}$ & 322 & 0.57 & 459 & 0.07 \\
\hline $\mathrm{In}_{2} \mathrm{O}_{3}$ & 454 & 1.50 & 542 & 1.37 \\
\hline $\mathrm{ZrO}_{2}$ & 440 & 1.31 & 534 & 1.94 \\
\hline $\mathrm{Ce}_{2} \mathrm{O}_{3}$ & 351 & 1.34 & 453 & 1.98 \\
\hline $\mathrm{MnO}_{2}$ & 379 & 2.58 & 403 & 2.55 \\
\hline $\mathrm{Co}_{3} \mathrm{O}_{4}$ & 375 & 1.70 & 396 & 2.81 \\
\hline $\mathrm{CuO}$ & 445 & 0.50 & 504 & 1.00 \\
\hline $\mathrm{Bi}_{2} \mathrm{O}_{3}$ & 425 & 0.79 & 510 & 0.38 \\
\hline $\mathrm{Sb}_{2} \mathrm{O}_{3}$ & 448 & 0.76 & 511 & 1.51 \\
\hline $\mathrm{TiO}_{2}$ & 485 & 1.41 & 527 & 1.22 \\
\hline $\mathrm{Al}_{2} \mathrm{O}_{3}$ & 511 & 3.73 & 526 & 2.87 \\
\hline $\mathrm{Nb}_{2} \mathrm{O}_{5}$ & 498 & 0.82 & 540 & 1.49 \\
\hline $\mathrm{Ta}_{2} \mathrm{O}_{5}$ & 528 & 1.16 & 541 & 1.21 \\
\hline $\mathrm{Ga}_{2} \mathrm{O}_{5}$ & 522 & 3.20 & 538 & 2.62 \\
\hline $\mathrm{Ge}_{2} \mathrm{O}_{5}$ & 510 & 1.38 & 536 & 1.38 \\
\hline
\end{tabular}

catalyst mass: $0.05 \mathrm{~g}$, soot mass: $0.005 \mathrm{~g}$; reactant conditions: $\mathrm{C}+0.042 \% \mathrm{NO}+4.4 \% \mathrm{O}_{2}+\mathrm{He}$, flow rate: $100 \mathrm{~mL} \cdot \mathrm{min}^{-1}$
氧化物对碳颗粒和 $\mathrm{NO}_{x}$ 同时去除具有较高的催化 活性. 这一结果表明, 在单一金属氧化物催化剂中, $\mathrm{Cr} 、 \mathrm{Mn} 、 \mathrm{Co}$ 和 $\mathrm{Ni}$ 的氧化物催化剂较有利于碳颗粒 和 $\mathrm{NO}_{x}$ 同时去除.

\section{2 接触方式对催化活性的影响}

从图 2 中还可以看到, 催化剂与碳颗粒之间的 接触方式对碳颗粒和 $\mathrm{NO}_{x}$ 去除有重要影响: (1) 在 “紧密接触”方式下的 $T_{10}$ 都比在“松散接触”方式的 $T_{10}$ 低, 并且大部分金属氧化物在“紧密接触”方式 下可明显降低 $T_{10} ;(2)$ 大部分金属氧化物在“松散 接触”方式下的 $V_{\mathrm{deNO} x}$ 比在“紧密接触”方式下的 $V_{\mathrm{deNO}}$ 大, 少部分金属氧化物 (如 $\mathrm{Al}_{2} \mathrm{O}_{3} 、 \mathrm{Ga}_{2} \mathrm{O}_{5} 、 \mathrm{SnO}_{2}$ ) 在 “松 散接触”方式下的 $V_{\mathrm{deNO}}$ 值明显比在“紧密接触”方式 下的 $V_{\mathrm{deNO}}$ 小; (3) Cr、Mn、Co 和Ni 的氧化物在“紧密 接触”方式下具有相对较低的 $T_{10}$ 和相对较大的 $V_{\mathrm{deNO}}$, 在“松散接触”方式下, $T_{10}$ 和 $V_{\mathrm{deN} O x}$ 的变化相对较小. 这些结果表明, 碳颗粒与催化剂之间“紧密接触”有 利于碳颗粒的氧化而几乎不利于 $\mathrm{NO}_{x}$ 减少, 但在 $\mathrm{Cr} 、 \mathrm{Mn} 、 \mathrm{Co}$ 和 $\mathrm{Ni}$ 的氧化物催化剂上接触方式对同 时催化去除碳颗粒和 $\mathrm{NO}_{x}$ 的影响变小.

\section{3 碳颗粒和 $\mathrm{NO}_{x}$ 同时去除路径分析}

对于碳颗粒与催化剂之间 “紧密接触” 有利于 碳颗粒的氧化, 这一结果与 Neeft 等 $\left.{ }^{\left[{ }^{[13]}\right.}\right]_{2}$ 道的碳颗 粒在空气中催化氧化的情况一致. 但值得注意的 是, 在 Neeft 等 ${ }^{[13]}$ 报道的结果中, $\mathrm{Cr} 、 \mathrm{Mn} 、 \mathrm{Co}$ 和 $\mathrm{Ni}$ 在 “松散接触”方式下对碳颗粒氧化几乎没有活性, 而 在本研究中, Cr、Mn、Co 和 $\mathrm{Ni}$ 在“松散接触”方式 下对碳颗粒氧化表现了较好的活性, 它们之间的差

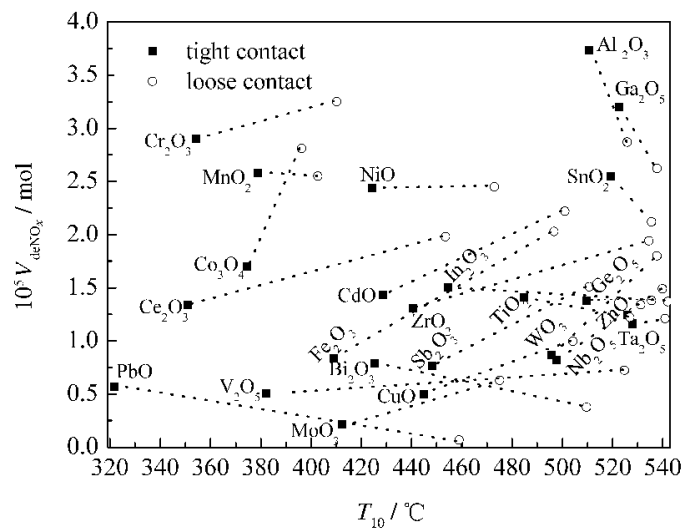

图 2 各催化剂与碳颗粒分别在“紧密接触”和“松散接触” 方式下同时去除碳颗粒和 $\mathrm{NO}_{x}$ 时 $\boldsymbol{T}_{10}$ 和 $\boldsymbol{V}_{\mathrm{deN} 0 x}$ 的相关性

Fig.2 Relation between $T_{10}$ and $V_{\mathrm{deNO} x}$ over various catalysts in tight and loose contact mode catalyst mass: $0.05 \mathrm{~g}$, soot mass: $0.005 \mathrm{~g}$; reactant conditions: $\mathrm{C}+0.042 \% \mathrm{NO}+4.4 \% \mathrm{O}_{2}+\mathrm{He}$, flow rate: $100 \mathrm{~mL} \cdot \mathrm{min}^{-1}$ 

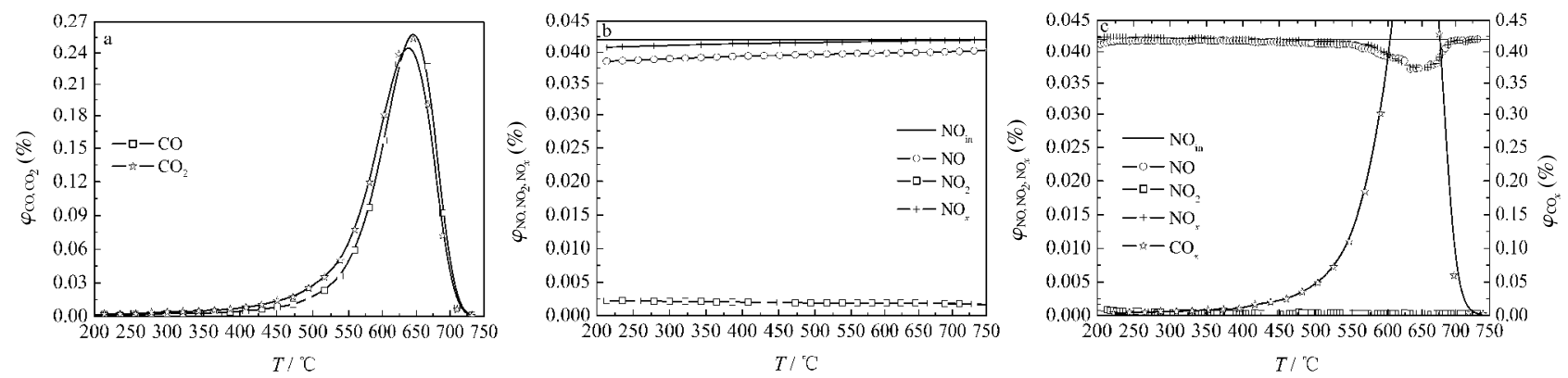

图 $3 \mathrm{SiO}_{2}$ 上不同反应条件的 TPR 曲线

Fig.3 TPR curves over $\mathrm{SiO}_{2}$ under different reactant conditions

(a) $\mathrm{C}+4.4 \% \mathrm{O}_{2}+\mathrm{He}$, (b) $0.042 \% \mathrm{NO}+4.4 \% \mathrm{O}_{2}+\mathrm{He}$, (c) $\mathrm{C}+0.042 \% \mathrm{NO}+4.4 \% \mathrm{O}_{2}+\mathrm{He}$; catalyst mass: $0.05 \mathrm{~g}$, soot mass: $0.005 \mathrm{~g}$, flow rate: $100 \mathrm{~mL} \cdot \mathrm{min}^{-1}$

异有待进行讨论. 另外, 对于碳颗粒与催化剂之间 “紧密接触” 几乎不利于 $\mathrm{NO}_{x}$ 减少, 其原因也有待进 一步讨论. 为此, 本研究对碳颗粒和 $\mathrm{NO}_{x}$ 同时去除 路径进行了分析.

图 3 为空白实验结果. 图 $3 \mathrm{a}$ 为 $\mathrm{SiO}_{2}$ 与碳颗粒 在“松散接触”方式下进行 $\mathrm{C}+\mathrm{O}_{2}$ 反应时 $\mathrm{CO}$ 和 $\mathrm{CO}_{2}$ 的 TPR 曲线. 从图 $3 \mathrm{a}$ 中可以看到, 碳颗粒氧化温 度很高 $\left(T_{10}=531{ }^{\circ} \mathrm{C}\right)$, 是典型的非催化氧化, 其反应式 如方程(1). 图 $3 \mathrm{~b}$ 为无碳颗粒存在条件下 $\mathrm{SiO}_{2}$ 上进 行 $\mathrm{NO}+\mathrm{O}_{2}$ 反应时 $\mathrm{NO} 、 \mathrm{NO}_{2}$ 和 $\mathrm{NO}_{x}$ 的 TPR 曲线. 从 图 $3 \mathrm{~b}$ 中可以看到, $\mathrm{NO} 、 \mathrm{NO}_{2}$ 和 $\mathrm{NO}_{\mathrm{x}}$ 的浓度几乎都 不发生变化, 表明 $\mathrm{SiO}_{2}$ 几乎不催化 $\mathrm{NO}$ 转化. 图 3c 为 $\mathrm{SiO}_{2}$ 与碳颗粒在 “松散接触”方式下进行 $\mathrm{C}+\mathrm{NO}+$ $\mathrm{O}_{2}$ 反应时 $\mathrm{NO} 、 \mathrm{NO}_{2} 、 \mathrm{NO}_{x}$ 和 $\mathrm{CO}_{x}$ 的 TPR 曲线. 从图 $3 \mathrm{c}$ 中可以看到, 碳颗粒氧化温度很高 $\left(T_{10}=539{ }^{\circ} \mathrm{C}\right)$, $\mathrm{NO}_{x}$ 减少与碳颗粒氧化发生在同一温度范围内. 将 图 3c 与图 3a 进行对照可以看到, 碳颗粒的氧化温 度几乎相同, 这表明 NO 的存在对碳颗粒氧化几乎 没有作用, 碳颗粒氧化主要仍归因于方程(1). 将图 $3 \mathrm{c}$ 与图 $3 \mathrm{~b}$ 进行对照可以看到, $\mathrm{NO}_{x}$ 减少明显, 并且 $\mathrm{NO}_{x}$ 减少的温度窗口与碳颗粒氧化的温度窗口一 致, 这表明 $\mathrm{NO}_{x}$ 减少与碳颗粒氧化相关. 由图 $3 \mathrm{c}$ 与
图 3a 的对照结果已知, 在该温度下 NO 与碳颗粒 直接发生氧化还原反应的可能性很小, 那么, $\mathrm{NO}_{x}$ 减少主要只能归因于碳颗粒氧化中间物 CO 对 $\mathrm{NO}$ 还原, 其反应式如方程(2). 这与 Yamashita 等 ${ }^{[15]}$ 报 道的结果一致.

$$
\begin{aligned}
& \mathrm{C}+\mathrm{O}_{2} \rightarrow \mathrm{CO}_{2} \text { (or } \mathrm{CO} \text { ) } \\
& \mathrm{CO}+\mathrm{NO} \rightarrow \mathrm{CO}_{2}+\mathrm{N}_{2}\left(\text { or } \mathrm{N}_{2} \mathrm{O}\right) \\
& \mathrm{CO}+\mathrm{O}_{2} \rightarrow \mathrm{CO}_{2} \\
& \mathrm{NO}+\mathrm{O}_{2} \rightarrow \mathrm{NO}_{2}
\end{aligned}
$$

图 4 为 $\mathrm{Co}_{3} \mathrm{O}_{4}$ 催化剂存在时的实验结果. 图 $4 \mathrm{a}$ 为 $\mathrm{Co}_{3} \mathrm{O}_{4}$ 与碳颗粒在 “松散接触”方式下进行 $\mathrm{C}+\mathrm{O}_{2}$ 反应时 $\mathrm{CO}$ 和 $\mathrm{CO}_{2}$ 的 TPR 曲线. 将图 $4 \mathrm{a}$ 与图 $3 \mathrm{a}$ 进 行对照可以看到, 碳颗粒氧化温度几乎相同 $\left(T_{10}=536\right.$ $\left.{ }^{\circ} \mathrm{C}\right)$, 但在 $\mathrm{Co}_{3} \mathrm{O}_{4}$ 上 $\mathrm{CO}$ 生成量明显降低, 表明 $\mathrm{Co}_{3} \mathrm{O}_{4}$ 对碳颗粒氧化无明显催化作用, 但能促进 $\mathrm{CO}$ 氧化, 其反应式如方程(3). 图 $4 \mathrm{~b}$ 为无碳颗粒存在条件下 $\mathrm{Co}_{3} \mathrm{O}_{4}$ 上进行 $\mathrm{NO}+\mathrm{O}_{2}$ 反应时 $\mathrm{NO} 、 \mathrm{NO}_{2}$ 和 $\mathrm{NO}_{x}$ 的 TPR 曲线. 将图 $4 \mathrm{~b}$ 与图 $3 \mathrm{~b}$ 进行对照可以看到, $\mathrm{Co}_{3} \mathrm{O}_{4}$ 上 $\mathrm{NO}$ 大部分被转化成 $\mathrm{NO}_{2}, \mathrm{NO}_{2}$ 的最大生成率为 $76 \%$, 其反应式如方程(4), 同时 $\mathrm{NO}_{x}$ 出现减少 $\left(V_{\mathrm{deNO}_{x}}=\right.$ $\left.1 \times 10^{-5} \mathrm{~mol}\right)$, 并且 $\mathrm{NO}_{x}$ 减少与 $\mathrm{NO}_{2}$ 形成相关, 这主 要由于 $\mathrm{NO}_{2}$ 的吸附分解 ${ }^{[16-18]}$ 导致. 图 $4 \mathrm{c}$ 为 $\mathrm{Co}_{3} \mathrm{O}_{4}$ 与
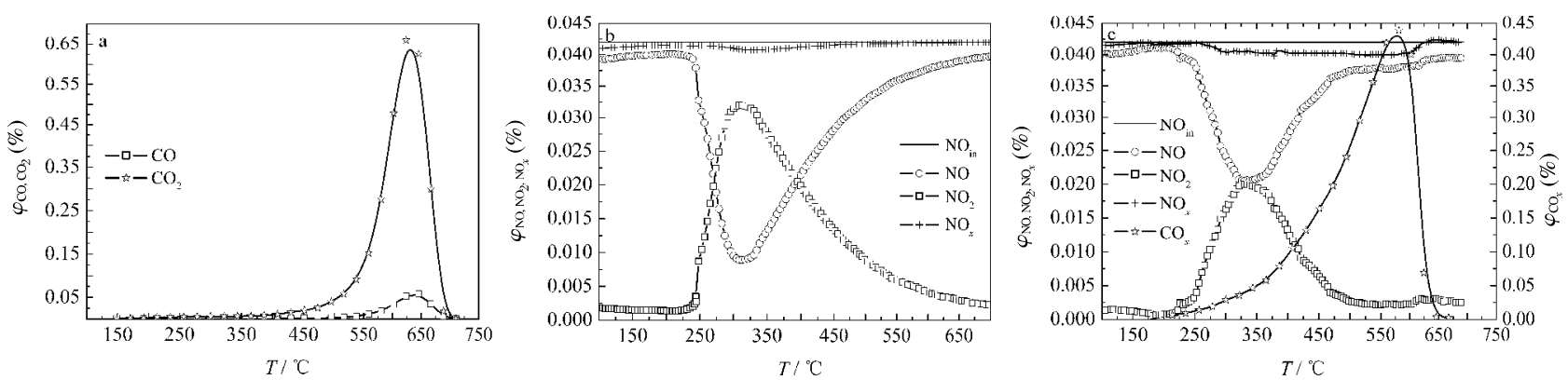

图 $4 \mathrm{Co}_{3} \mathrm{O}_{4}$ 上不同反应条件的 TPR 曲线

Fig.4 TPR curves over $\mathrm{Co}_{3} \mathrm{O}_{4}$ under different reactant conditions

(a) $\mathrm{C}+4.4 \% \mathrm{O}_{2}+\mathrm{He}$, (b) $0.042 \% \mathrm{NO}+4.4 \% \mathrm{O}_{2}+\mathrm{He}$, (c) $\mathrm{C}+0.042 \% \mathrm{NO}+4.4 \% \mathrm{O}_{2}+\mathrm{He}$; catalyst mass: $0.05 \mathrm{~g}$, soot mass: $0.005 \mathrm{~g}$, flow rate: $100 \mathrm{~mL} \cdot \mathrm{min}^{-1}$ 
碳颗粒在 “松散接触”方式下进行 $\mathrm{C}+\mathrm{NO}+\mathrm{O}_{2}$ 反应 时 $\mathrm{NO} 、 \mathrm{NO}_{2} 、 \mathrm{NO}_{x}$ 和 $\mathrm{CO}_{x}$ 的TPR 曲线. 将图 4c 与图 $4 \mathrm{~b}$ 对照可以看到, $\mathrm{NO}_{2}$ 几乎出现在同一温度, 但在 图 $4 \mathrm{c}$ 中 $\mathrm{NO}_{2}$ 生成浓度明显降低, $\mathrm{NO}$ 浓度明显升 高; 而将图 4c 与图 4a 对照可以看到, 在图 4c 中 $\mathrm{CO}_{x}$ 出现温度大幅降低 $\left(T_{10}=396{ }^{\circ} \mathrm{C}\right)$, 并且与 $\mathrm{NO}_{2}$ 出 现温度相同, 这些结果表明形成的 $\mathrm{NO}_{2}$ 将碳颗粒 氧化成 $\mathrm{CO}$ 和 $\mathrm{CO}_{2}$, 同时 $\mathrm{NO}_{2}$ 又还原成 $\mathrm{NO}$, 主要发 生了方程(5)的反应 ${ }^{[19]}$. 将图 $4 \mathrm{c}$ 与图 $4 \mathrm{~b}$ 对照还可以 看到, $\mathrm{NO}_{x}$ 减少总量增加 $\left(V_{\mathrm{deNO}_{x}}=2.8 \times 10^{-5} \mathrm{~mol}\right)$, 并且 $\mathrm{NO}_{x}$ 减少的温度窗口与碳颗粒氧化的温度窗口一 致, 这表明 $\mathrm{NO}_{x}$ 减少主要与碳颗粒氧化相关. 由于 在 $\mathrm{Co}_{3} \mathrm{O}_{4}$ 上碳颗粒被 $\mathrm{O}_{2}$ 氧化几乎在 $500{ }^{\circ} \mathrm{C}$ 以上才 发生(见图 4a), 当温度低于 $500{ }^{\circ} \mathrm{C}$ 时, $\mathrm{NO}_{x}$ 减少主要 归因于碳颗粒与 $\mathrm{NO}_{2}$ 的直接反应, 其反应式如方程 (6); 当温度高于 $500{ }^{\circ} \mathrm{C}$ 时, $\mathrm{NO}_{x}$ 减少还包括方程(2).

$$
\begin{aligned}
& \mathrm{NO}_{2}+\mathrm{C} \rightarrow \mathrm{CO}_{2}(\text { or } \mathrm{CO})+\mathrm{NO} \\
& \mathrm{NO}_{2}+\mathrm{C} \rightarrow \mathrm{CO}_{2}(\text { or } \mathrm{CO})+\mathrm{N}_{2}\left(\text { or } \mathrm{N}_{2} \mathrm{O}\right)
\end{aligned}
$$

由以上反应路径分析可知道, 在 $\mathrm{O}_{2}$ 存在条件 下碳颗粒和 $\mathrm{NO}_{x}$ 能够同时去除, 碳颗粒氧化的中 间物 $\mathrm{CO}$ 对 $\mathrm{NO}_{x}$ 减少起着重要的作用, 而 $\mathrm{NO}_{2}$ 的 生成能促进碳颗粒氧化. 考虑到方程(6)也包括中 间物 CO 的生成过程 ${ }^{[20]}$, 那么, 可将方程(6)写成方 程(7)的形式. 由此可见, 催化碳颗粒和 $\mathrm{NO}_{x}$ 同时去 除的路径为, 催化剂催化 $\mathrm{NO}$ 氧化成 $\mathrm{NO}_{2}, \mathrm{NO}_{2}$ 氧 化碳颗粒, 促进碳颗粒去除; 而碳颗粒氧化的中间 物 $\mathrm{CO}$ 还原 $\mathrm{NO}$, 促进 $\mathrm{NO}$ 去除.

$$
\mathrm{NO}_{2}+\mathrm{C} \rightarrow \mathrm{CO}+\mathrm{NO} \rightarrow \mathrm{CO}_{2}+\mathrm{N}_{2}\left(\text { or } \mathrm{N}_{2} \mathrm{O}\right)
$$

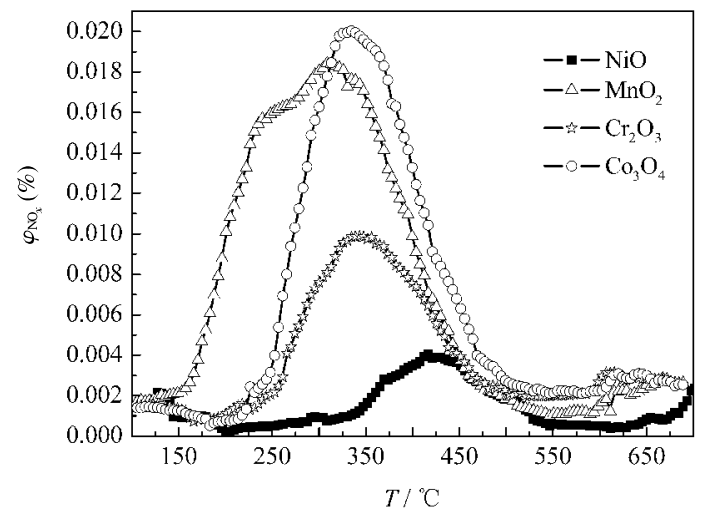

图 $5 \mathrm{Cr} 、 \mathrm{Mn} 、 \mathrm{Co}$ 和 $\mathrm{Ni}$ 的氧化物分别与碳颗粒在“松散接 触”方式下进行 $\mathrm{C}+\mathrm{NO}+\mathrm{O}_{2}$ 反应时生成 $\mathrm{NO}_{2}$ 的 TPR 曲线

Fig.5 TPR curves of $\mathrm{NO}_{2}$ over $\mathrm{Cr}_{2} \mathrm{O}_{3}, \mathrm{MnO}_{2}, \mathrm{Co}_{3} \mathrm{O}_{4}$ and $\mathrm{NiO}$ in loose contact mode

catalyst mass: $0.05 \mathrm{~g}$, soot mass: $0.005 \mathrm{~g}$; reactant conditions: $\mathrm{C}+0.042 \% \mathrm{NO}+4.4 \% \mathrm{O}_{2}+\mathrm{He}$, flow rate: $100 \mathrm{~mL} \cdot \mathrm{min}^{-1}$
依据以上反应路径知道, 碳颗粒在 $\mathrm{Co}_{3} \mathrm{O}_{4}$ 上低 温氧化主要归因于 $\mathrm{NO}_{2}$. 图 5 为 $\mathrm{Cr} 、 \mathrm{Mn} 、 \mathrm{Co}$ 和 $\mathrm{Ni}$ 的氧化物分别与碳颗粒在 “松散接触”方式下进行 $\mathrm{C}+\mathrm{NO}+\mathrm{O}_{2}$ 反应时生成 $\mathrm{NO}_{2}$ 的 TPR 曲线. 从图 5 中 可以看到, 在这些催化剂上都有较大量的 $\mathrm{NO}_{2}$ 生 成. 由于 $\mathrm{NO}_{2}$ 为流动的强氧化剂 ${ }^{[19,21,22]}$, 促进了碳颗 粒在“松散接触”方式下的氧化, 使接触方式对碳颗 粒氧化的影响变小, 从而导致在 $\mathrm{Cr} 、 \mathrm{Mn} 、 \mathrm{Co}$ 和 $\mathrm{Ni}$ 的氧化物催化剂上接触方式对同时催化去除碳颗 粒和 $\mathrm{NO}_{x}$ 的影响变小.

同样依据以上反应路径知道, $\mathrm{NO}_{x}$ 减少主要与 碳颗粒氧化中间物 $\mathrm{CO}$ 相关. 为了研究 $\mathrm{CO}$ 与 $\mathrm{NO}_{x}$ 减少的相关性, 本研究也计算了反应过程中生成 $\mathrm{CO}$ 的选择性 $\left(S_{\mathrm{CO}}\right)$ : 一个 $\mathrm{TPR}$ 过程中形成的总 $\mathrm{CO}$ 与 形成的总 $\mathrm{CO}_{x}$ 的比值. 图 6 为各催化剂与碳颗粒分 别在“紧密接触”和“松散接触”方式下进行 $\mathrm{C}+\mathrm{NO}+$ $\mathrm{O}_{2}$ 反应时的 $S_{\mathrm{CO}}$. 从图 6 中可以看到, 大部分金属 氧化物在“松散接触”方式下的 $S_{\mathrm{CO}}$ 比在“紧密接 触”方式下的 $S_{\mathrm{CO}}$ 大. 由于 “松散接触”方式更利于 $\mathrm{CO}$ 生成, 从而导致了 “松散接触” 方式下更利于 $\mathrm{NO}_{x}$ 减少. 对于少数金属氧化物催化剂 (如 $\mathrm{Al}_{2} \mathrm{O}_{3}$ 、 $\mathrm{Ga}_{2} \mathrm{O}_{5} 、 \mathrm{SnO}_{2}$ ) 在“松散接触”方式下的 $V_{\text {deNO }}$ 明显小于 在“紧密接触”方式下的 $V_{\mathrm{deNO}_{x}}$, 它们的 $V_{\mathrm{deNO}}$ 与 $S_{\mathrm{CO}}$ 不一定有直接的对应关系, 但这些催化剂主要是催 化碳颗粒氧化活性较差的催化剂, 其与碳颗粒的接 触方式对碳颗粒氧化影响很小, 在两种接触方式下 碳颗粒氧化情况相近, 进行 $\mathrm{C}+\mathrm{O}_{2}$ 反应时产生的 $\mathrm{CO}$ 浓度几乎相同. 在几乎相同的 CO 浓度条件下, “紧

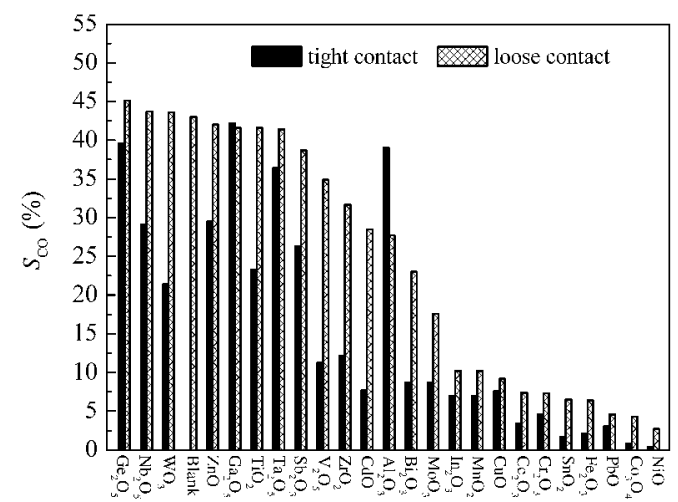

图 6 催化剂与碳颗粒分别在“紧密接触”和“松散接触”方 式下进行 $\mathrm{C}+\mathrm{NO}+\mathrm{O}_{2}$ 反应时生成 $\mathrm{CO}$ 的选择性

Fig.6 Selectivity to produce $\mathrm{CO}$ over various catalysts in tight and loose contact mode catalyst mass: $0.05 \mathrm{~g}$, soot mass: $0.005 \mathrm{~g}$; reactant conditions: $\mathrm{C}+0.042 \% \mathrm{NO}+4.4 \% \mathrm{O}_{2}+\mathrm{He}$, flow rate: $100 \mathrm{~mL} \cdot \mathrm{min}^{-1}$ 
密接触”方式可能更有利于 $\mathrm{NO}_{x}$ 减少.

\section{3 结 论}

在单一金属氧化物催化剂中, $\mathrm{Cr} 、 \mathrm{Mn} 、 \mathrm{Co}$ 和 $\mathrm{Ni}$ 金属氧化物催化剂对碳颗粒和 $\mathrm{NO}_{x}$ 同时去除具有 较高的催化活性, 其催化同时去除碳颗粒和 $\mathrm{NO}_{x}$ 的路径为: 催化剂催化 $\mathrm{NO}$ 氧化成 $\mathrm{NO}_{2}, \mathrm{NO}_{2}$ 促进 碳颗粒氧化去除, 而碳颗粒氧化的中间物 $\mathrm{CO}$ 还原 $\mathrm{NO}$, 促进 NO 还原去除. 催化剂与碳颗粒之间的接 触方式对碳颗粒氧化和 $\mathrm{NO}_{x}$ 还原有重要的影响, 在“紧密接触”方式下有利于碳颗粒氧化, 在 “松散 接触”方式下有利于 $\mathrm{NO}_{x}$ 还原, 但当催化剂有利于 促进强氧化剂 $\mathrm{NO}_{2}$ 形成时, 接触方式对催化同时 去除碳颗粒和 $\mathrm{NO}_{x}$ 的影响变小.

\section{References}

1 Yoshida, K.; Makino, S.; Sumiya, S.; Muramatsu, G.; Helferich, R. Simultaneous reduction of $\mathrm{NO}_{x}$ and particulate emissions from diesel engine exhaust. SAE paper, 892046. 1989

2 Teraoka, Y.; Nakano, K.; Shangguan, W. F.; Kagawa, S. Catal. Today, 1996, 27: 107

3 Shangguan, W. F.; Teraoka, Y.; Kagawa, S. Appl. Catal. B, 1996, 8: 217

4 Liu, Z. M.; Hao, Z. P.; Guo, Y.; Zhuang, Y. H. J. Envir. Sci., 2002, 14: 289

5 Liu, G. H.; Huang, Z.; Shangguan, W. F.; Yan, C. X. Chin. Sci. Bull., 2002, 47: 1620 [刘光辉, 黄 震, 上官文峰, 阎存仙. 科学通报, 2002, 47: 1620]

6 Kureti, S.; Weisweiler, W.; Hizbullah, K. Appl. Catal. B, 2003, 43:
281

7 Pei, M. X.; Lin, H.; Shangguan, W. F.; Huang, Z. Acta Phys. Chim. Sin., 2005, 21: 255 [裴梅香, 林 赫, 上官文峰, 黄 震. 物理化学学报, 2005, 21: 255]

8 Fino, D.; Russo, N.; Saracco, G.; Specchia, V. J. Catal., 2006, 242: 38

9 Castoldi, L.; Matarrese, R.; Lietti, L.; Forzatti, P. Appl. Catal. B, 2006, 64: 25

10 Peng, X. S.; Lin, H.; Shangguan, W. F.; Huang, Z. Catal. Commun., 2007, 8: 157

11 Liu, J.; Zhao, Z.; Xu, C. M.; Duan, A. J.; Meng, T.; Bao, X. J. Catal. Today, 2007, 119: 267

12 Zhu, R. S.; Guo, M. X.; Ci, X. B.; Ouyang, F. Catal. Commun., 2008, 9: 1184.

13 Neeft, J. P. A.; Makkee, M.; Moulijn, J. A. Appl. Catal. B, 1996, 8: 57

14 Zhu, R. S.; Guo, M. X.; Ouyang, F. Acta Phys. -Chim. Sin., 2008, 24: 909 [朱荣淑, 郭明新, 欧阳峰. 物理化学学报, 2008, 24: 909]

15 Yamashita, H.; Tomita, A. Energy Fuels, 1993, 7: 85

16 Frizt, A.; Pitchon, V. Appl. Catal. B, 1997, 13: 1

17 Winter, E. R. S. J. Catal., 1971, 22: 158

18 Li, Y. J.; Hall, W. K. J. Catal., 1991, 129: 202

19 Liu, S. T.; Obuchi, A.; Oi-Uchisawa, J.; Nanba, T.; Kushiyama, S. Appl. Catal. B, 2001, 30: 259

20 Shangguan, W. F.; Teraoka, Y.; Kagawa, S. Appl. Catal. B, 1997, 12: 237

21 Oi-Uchisawa, J.; Obuchi, A.; Ogata, A.; Enomoto, R.; Kushiyama, S. Appl. Catal. B, 1999, 21: 9

22 Oi-Uchisawa, J.; Obuchi, A.; Enomoto, R.; Xu, J. Y.; Nanba, T.; Liu, S. T.; Kushiyama, S. Appl. Catal. B, 2001, 32: 257 